Ricard Ferrer

María Carmen Fariñas ${ }^{2}$

Emilio Maseda $^{3}$

Miguel Salavert ${ }^{4}$

German Bou ${ }^{5}$

Jazmín Díaz-Regañón ${ }^{6}$

Diego López ${ }^{6}$

Virginia Lozano ${ }^{6}$

David Gómez-Ulloa

Raquel Fenoll

Nuria Lara $^{7}$

Eilish McCann ${ }^{8}$

\title{
Clinical management of cUTI, cIAl, and HABP/VABP attributable to carbapenem-resistant Gram-negative infections in Spain
}

\author{
${ }^{1}$ Hospital Universitario Vall d'Hebron, Barcelona, Spain. \\ ${ }^{2}$ Hospital Marqués de Valdecilla, Santander, Spain. \\ ${ }^{3}$ Hospital Universitario La Paz, Madrid, Spain. \\ ${ }^{4}$ Hospital Universitario y Politécnico La Fe, Valencia, Spain. \\ ${ }^{5}$ Hospital Universitario A Coruña, La Coruña, Spain. \\ ${ }^{6}$ MSD, Madrid, Spain. \\ ${ }^{7}$ IOVIA, Barcelona, Spain. \\ ${ }^{8}$ Merck \& Co., Inc., Kenilworth NJ, USA.
}

Article history

Received: 29 June 2021; Revision Requested: 19 July 2021; Revision Received: 30 August 2021; Accepted: 2 September 2021; Published: 22 November 2021

\section{ABSTRACT}

Introduction. Carbapenem-resistant Gram-negative (CRGN) infections are a major public health problem in Spain, often implicated in complicated, healthcare-associated infections that require the use of potentially toxic antibacterial agents of last resort. The objective of this study was to assess the clinical management of complicated infections caused by CRGN bacteria in Spanish hospitals.

Methods. The study included: 1) a survey assessing the GN infection and antibacterial susceptibility profile in five participating Spanish hospitals and 2) a non-interventional, retrospective single cohort chart review of 100 patients with complicated urinary tract infection (cUTI), complicated intra-abdominal infection (cIAl), or hospital-acquired bacterial pneumonia/ventilator-associated bacterial pneumonia (HABP/ VABP) attributable to CRGN pathogens.

Results. In the participating hospitals CRGN prevalence was 9.3\% amongst complicated infections. In the retrospective cohort, 92\% of infections were healthcare-associated, and Klebsiella pneumoniae and Pseudomonas aeruginosa were the most common pathogens. OXA was the most frequently detected carbapenemase type (71.4\%). We found that carbapenems were frequently used to treat cUTI, cIAI, HABP/VABP caused by CRGN pathogens. Carbapenem use, particularly in combination with other agents, persisted after confirmation of carbapenem resistance. Clinical cure was $66.0 \%$, mortality during hospitalization $35.0 \%$, mortality at the time of chart review 62.0\%, and 6-months-post-discharge readmission 47.7\%.

Conclusion. Our results reflect the high burden and unmet needs associated with the management of complicated infections attributable to CRGN pathogens in Spain and high-

Correspondence:

Dr. Ricard Ferrer

Hospital Universitario Vall d'Hebron, Barcelona, Spain.

Phone: 934893000

E-mail: r.ferrer@vhebron.net light the urgent need for enhanced clinical management of these difficult-to-treat infections.

Keywords: Carbapenem-resistant Gram-negative infections; Carbapenemase; Carbapenems; Healthcare-associated infections; Antimicrobial stewardship.

\section{Manejo clínico de las ITUc, IIAc y NAH/NAVM atribuidas a bacterias gramnegativas resistentes a carbapenémicos en España}

\section{RESUMEN}

Introducción. Las infecciones por bacterias gramnegativas resistentes a carbapenémicos (GNRC) son un problema sanitario en España, pues suelen estar implicadas en infecciones complicadas relacionadas con la asistencia sanitaria, que requieren antibacterianos potencialmente tóxicos como último recurso. El objetivo fue evaluar el tratamiento de infecciones complicadas causadas por bacterias GNRC en hospitales españoles.

Métodos. El estudio incluyó, una encuesta sobre la infección por gramnegativas y el perfil de sensibilidad antibacteriana en cinco hospitales españoles y una revisión no intervencionista retrospectiva de historias clínicas de una cohorte de 100 pacientes con infección urinaria complicada (ITUc), infección intrabdominal complicada (IIAC) o neumonía bacteriana adquirida en el hospital o asociada a ventilación mecánica (NAH/ NAVM) causadas por patógenos GNRC.

Resultados. En hospitales españoles, la prevalencia de bacterias GNRC fue del 9,3\% entre las infecciones complicadas. En la cohorte retrospectiva, el $92 \%$ de las infecciones estaban relacionadas con la asistencia sanitaria y los patógenos más comunes fueron Klebsiella pneumoniae (51\%) y Pseudomonas aeruginosa (44\%). La carbapenemasa más frecuente fue OXA (71,4\%). Observamos que los carbapenémicos se usaban frecuentemente para tratar las ITUc, IIAc y NAH/NAVM 
causadas por bacterias GNRC. Su uso, especialmente en combinación, se mantuvo aún después de la confirmación de la resistencia. La curación fue del 66,0\%, la mortalidad durante la hospitalización del $35,0 \%$, la mortalidad total del $62,0 \%$ y el reingreso 6 meses después del alta del 47,7\%.

Conclusión. Nuestros resultados reflejan la elevada carga sanitaria y las necesidades no cubiertas asociadas al tratamiento de infecciones complicadas por bacterias GNRC en España y evidencian la urgencia de contar con nuevas alternativas para su tratamiento.

\section{INTRODUCTION}

Antimicrobial resistance (AMR) is one of modern healthcare's most difficult challenges, associated with 1,600 million $€$ in healthcare costs, 2.5 million additional hospital days, and 25,000 deaths yearly in the European Union alone [1-3]. Healthcare-associated infections, often caused by the ESKAPE pathogens (Enterococcus faecium, Staphylococcus aureus, Klebsiella pneumoniae, Acinetobacter baumannii, Pseudomonas aeruginosa, and Enterobacterales spp.), are especially prone to multidrug resistance, limiting therapeutic options.

Carbapenems are high-efficacy, well-tolerated antibacterial agents, used frequently as a treatment for complicated infections [4]. The emerging resistance of Gram-negative (GN) pathogens to carbapenems is therefore particularly alarming. The most common carbapenem resistance types are the carbapenemase (KPC), the New Delhi metallo-beta-lactamase (NDM), the Verona Integron-Mediated (VIM) metallo-beta-lactamase, or the OXA-48 family of carbapenemases [1]. Carbapenem-resistant GN (CRGN) pathogens are often associated with complicated, healthcare-associated infections for which clinicians may have to use potentially toxic antibacterial agents of last resort, such as colistin, or synergistic therapeutic combinations of questionable effectiveness, due to the ineffectiveness of carbapenems [5]. The situational necessity to make treatment decisions before the availability of microbial confirmation or antibacterial susceptibility results, the potential negative consequences of choosing an inappropriate empirical therapy, and the complex antibacterial switching and add-on behavior following patient deterioration or receipt of results further complicates the antimicrobial stewardship picture for these infections [4].

Surveillance data indicate that CRGN infections are more prevalent in southern European countries, with the potential emergence of pan-resistant strains of certain pathogens [2]. In Spain, 3.2\% of healthcare-associated infections are caused by CRGN pathogens [6] and a widespread increase of empirical carbapenem use in Spanish hospitals has been observed [7]. As such, CRGN infections are a major public health problem for the Spanish National Health Service, responsible for an estimated €472 million in healthcare costs in 2017 [6].

The conditions associated with CRGN infections that are of most concern include complicated urinary tract infections (cUTI) associated with catheter use, surgery-associated com- plicated intra-abdominal infections (cIAI), and hospital-acquired bacterial pneumonia and ventilator-associated bacterial pneumonia (HABP/NABP) - the latter being highly common in intensive care units (ICU) [8]. A better understanding of the clinical characteristics and current therapeutic management of the patient population affected by these infections is urgently needed in order to identify suboptimal treatment options and potential new treatment strategies to help improve clinical and economic outcomes and to facilitate improved antimicrobial stewardship. The objective of this study was to characterize the real-world clinical management and outcomes of patients with cUTI, cIAl, or HABP/NABP caused by CRGN pathogens in Spanish hospitals.

\section{METHODS}

The EU-CARE is an observational study of the clinical management of CUTI, CIAI, and HABP/NABP attributable to CRGN infections. The study included: 1) a survey assessing the GN infection and antibacterial susceptibility profile of the participating hospitals, and 2) a non-interventional, retrospective single cohort chart review in the participating hospitals of patients with CUTI, CIAI, or HABP/NABP attributable to CRGN pathogens. Throughout the study, carbapenem resistance was defined as per the European Committee on Antimicrobial Susceptibility Testing (EUCAST) screening cut-off values based on the EUCAST criteria in use at time of culture collection (2017) [9]. Resistant or intermediate isolates were defined as being non-susceptible, i.e. carbapenem-resistant for the purposes of study inclusion.

Hospital survey. Participating hospitals were a convenience sample of five large (>600 bed) teaching hospitals in Spain. The survey included an assessment of the number of CRGN infections and the percentage and type of carbapenem non-susceptibility by infection site as observed between 2015 year and 2018 year. Of these CRGN infections the percentage of isolates non-susceptible to carbapenem(s) and other antibacterial agents was reported. The pathogens specifically assessed were: Escherichia coli, K. pneumoniae, other Enterobacterales, $P$. aeruginosa, and A. baumannii. The participating hospitals served as the sampling frame for the patient chart review study.

Retrospective follow-up chart review study of patients. Investigators in the above hospitals were physicians experienced in managing complicated infections. They retrospectively examined patients' medical records for a 30-month period (patient identification window period), starting from approximately July 2017 and going back in time until a total number of 100 patients were identified across the participating hospitals. The follow-up period for each patient ranged from the culture collection date to either 6 months after hospital discharge date or the date of death, whichever occurred first, and therefore varied from patient to patient depending on the duration of the hospital admission from the point of culture collection. 
The inclusion criteria required patients to: be at least 18 years of age, have a hospital admission date within the patient identification window period, have a physician confirmed diagnosis of $\mathrm{CUTI}, \mathrm{clAl}$, or HABP/VABP (infection opposed to colonization i.e. with signs and symptoms of clinical infection) attributable to CRGN pathogens (as per EUCAST screening cutoff values) based on the culture(s) collected during the index hospitalization, and be available medical data from culture collection to the end of follow-up. Patients participating in a clinical trial during the observation period were excluded.

Variables assessed. The chart review assessed patient characteristics, index hospitalization characteristics, infection characteristics, antibiogram results, carbapenemase testing procedures, patient outcomes, treatment pathways, and healthcare resource utilization during the index hospitalization. Patient characteristics included age, sex, body mass index (BMI), smoking behavior, alcohol consumption, comorbidities, hypersensitivity to antibacterial agents, reason for admission, and ward of admission. In addition, the year prior to hospitalization was assessed to determine whether patients had been admitted to a hospital, been in a nursing home, undergone invasive procedures, or had been administered antibacterial agents. Index hospitalization characteristics included - disease severity reported as Acute Physiology And Chronic Health Eval- uation (APACHE II) score and overall and ICU length of stay (LOS) reported for the overall sample and stratified by infection site. Overall LOS was defined as the start date of a hospital admission until a patient's discharge or death; whichever occurred first. Presence in the ICU, and thus ICU LOS, was defined as the number of days that the patient remained in ICU during the index hospitalization.

Infection characteristics included infection site, presence of secondary bacteremia, place of acquiring infection (hospital-, healthcare- or community-acquired), pathogen isolated, pathogen verification method, antibacterial susceptibility verification method and results, and the sample type from which the culture was sourced. Carbapenemase testing procedures were reported by the hospital, and included procedures performed to identify carbapenemase production and to determine carbapenemase type.

Outcomes included whether clinical cure (i.e. the complete resolution of all signs and symptoms of infection) was achieved and the time to clinical cure (from the date of culture collection, defined as the date the bacterial culture confirming the diagnosis of cUTI, cIAI, or HABP/NABP caused by CRGN pathogens was collected and sent for testing). Additional outcome variables were occurrence and duration of severe sepsis/septic shock, mortality during index hospitalization and during the follow-up period, and time to discharge after culture collection.
A

Number of infections and susceptibility

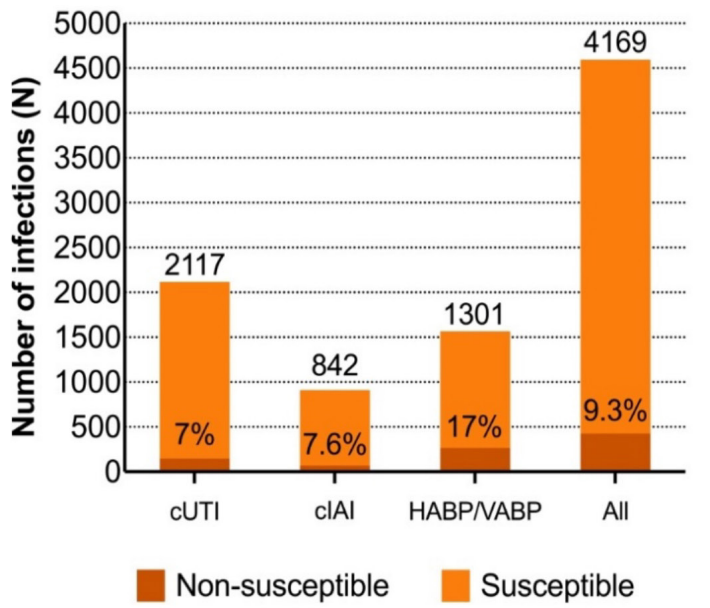

B

\section{Carbapenemase resistance types}

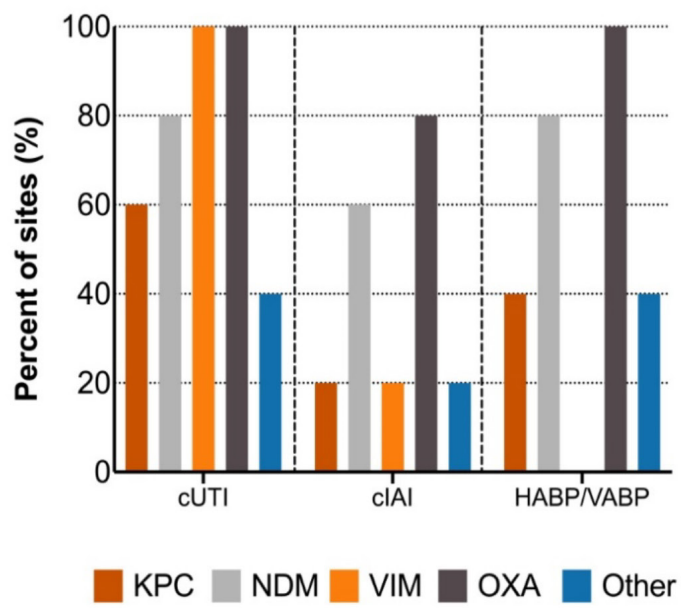

Figure $1 \quad$ Hospital-reported Gram-negative infection and carbapenem susceptibility characteristics. Figure $1 \mathrm{~A}$ shows mean number of infections; colors represent the proportion of isolates which are susceptible/non-susceptible. Figure 1B shows percentage of hospital sites that reported different types of carbapenemase resistance by infection site.

Data based on initial assessment performed by the sites $(n=5)$ regarding the number and type of complicated gram-negative infections over the last 3 years.

cIAl: Complicated intra-abdominal infection; cUTI: Complicated urinary tract infection; GN: Gram-negative; HABP/VABP: Hospital-acquired bacterial pneumonia/ventilator-associated bacterial pneumonia; KPC: Klebsiella pneumoniae carbapenemase; NDM: New Delhi metallo-beta-lactamase; OXA: Oxacillinase type; VIM: Verona Integron-Mediated metallo-beta-lactamase. 


\section{Carbapenem resistance}

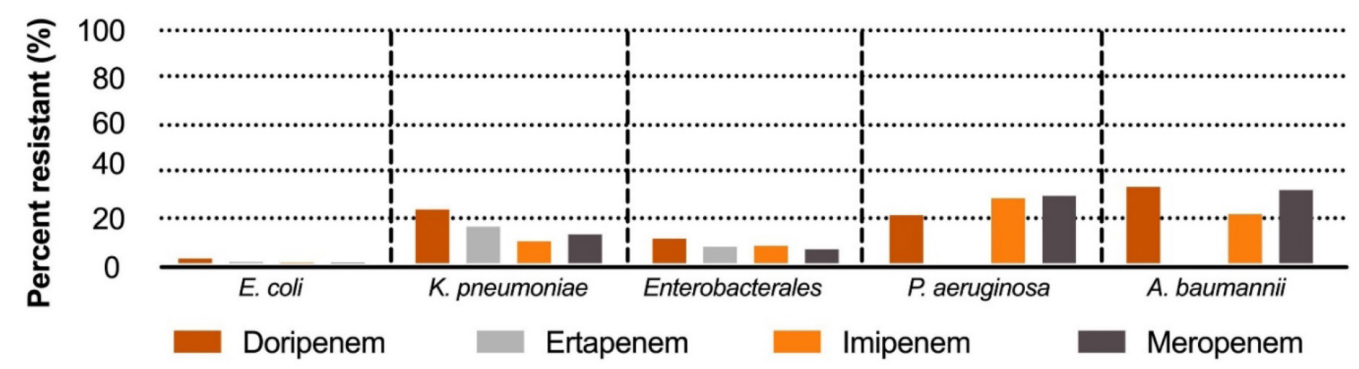

Other antibacterial resistance

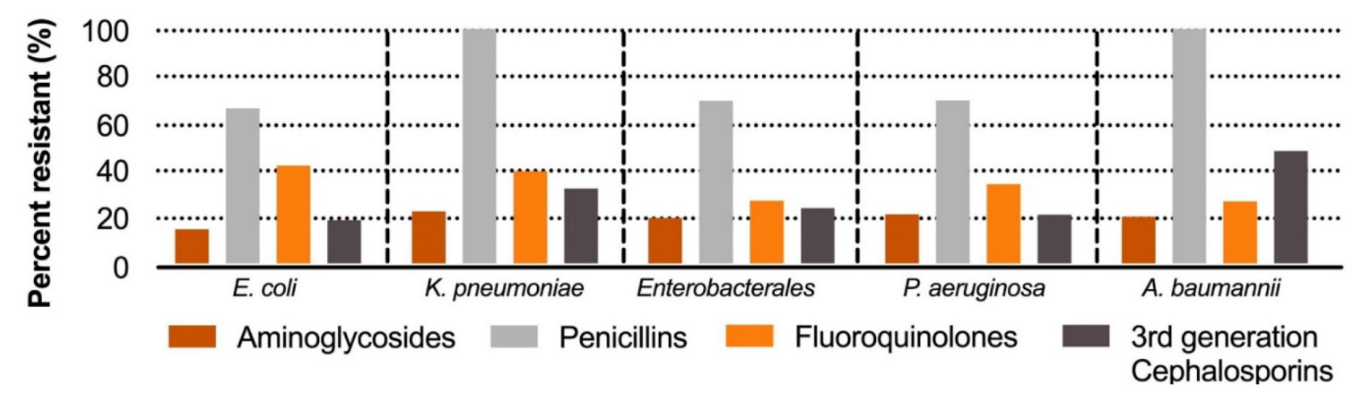

Figure 2 Antibacterial resistance by bacterial type. Percentage of isolates that were resistant to each antibacterial class in 2017

Percent resistance refers to the percent non-susceptible (intermediate or resistant). Ertapenem resistance is not shown for Pseudomonas and Acinetobacter isolates, since these pathogens are intrinsically resistant to this antibacterial agent.

Ethics. The study protocol was originally approved by the Clinical Research Ethics Committee of Hospital Universitari Vall d'Hebron (CEIm - Hospital Universitari Vall d'Hebron, de BarceIona, Spain approval number MER-ANT-2017-01). The rest of participant centres ratified the original approval.

Due to the secondary nature of the data, the need for patient informed consent was waived by the reference IECS.

Data analysis. Statistical analyses were conducted with SAS Enterprise Guide version 7.13. Study objectives were addressed using descriptive statistics. Continuous variables were described with mean and standard deviation (SD) for patients with valid observations, and categorical variables with the number and percentage of patients in each category. Furthermore, a subset analysis was performed on patients receiving carbapenems at any timepoint during index hospitalization $(n=57)$ in order to assess their treatment pathways. Missing data were not substituted or included in the calculations due to the real-world nature of the analysis, except for missing dates, which were replaced by middle of the month dates.

\section{RESULTS}

GN infection and antibacterial susceptibility profile of the participating hospitals. Hospitals reported CUTI as the most common infection site, followed by HABP/NABP and
clAl (Figure 1A). Of all GN infections, 9.3\% were reported to be carbapenem non-susceptible (7.0\% of cUTI, 7.6\% of cIAI, and $17.0 \%$ of HABP/VABP isolates). The mechanism for carbapenem resistance was most diverse for cUTI (highest number of hospital sites reporting the most carbapenemase types), whereas for clAI and HABP/NABP isolates the carbapenemase type was predominantly NDM and OXA-type (Figure 1B).

$P$. aeruginosa and $A$. baumannii isolates showed the highest level of multi-carbapenem resistance: over 20\% of these bacterial isolates were resistant to all four carbapenems (Figure 2A). Similarly, over $20 \%$ of $K$. pneumoniae, $P$. aeruginosa, and $A$. baumannii showed resistance to all other antibacterial agents, and over $60 \%$ of all pathogens were resistant to penicillin (Figure 2B).

Patient characteristics. Table 1 shows the characteristics of participating patients. In summary, patients were predominantly male (70\%), overweight (BMI $\left.>25.0 \mathrm{~kg} / \mathrm{m}^{2}: 40 \%\right)$, and elderly (mean age: 65.9 [SD=14.5] years). Prior hospital admission was common (58\%). Over half of patients had had at least one invasive procedure or had been treated with antibacterial agents (mostly penicillins [67.3\%, $n=35]$ and fluroquinolones $[51.9 \%, n=27])$ in the year prior to admission. Comorbidities were also common (88.0\%), especially hypertension (39.0\%), chronic lung disease $(26.0 \%)$, and diabetes $(26.0 \%)$. The mean LOS was 70.3 ( $S D=34.2$ ) days (Figure 3A). Half of patients re- 


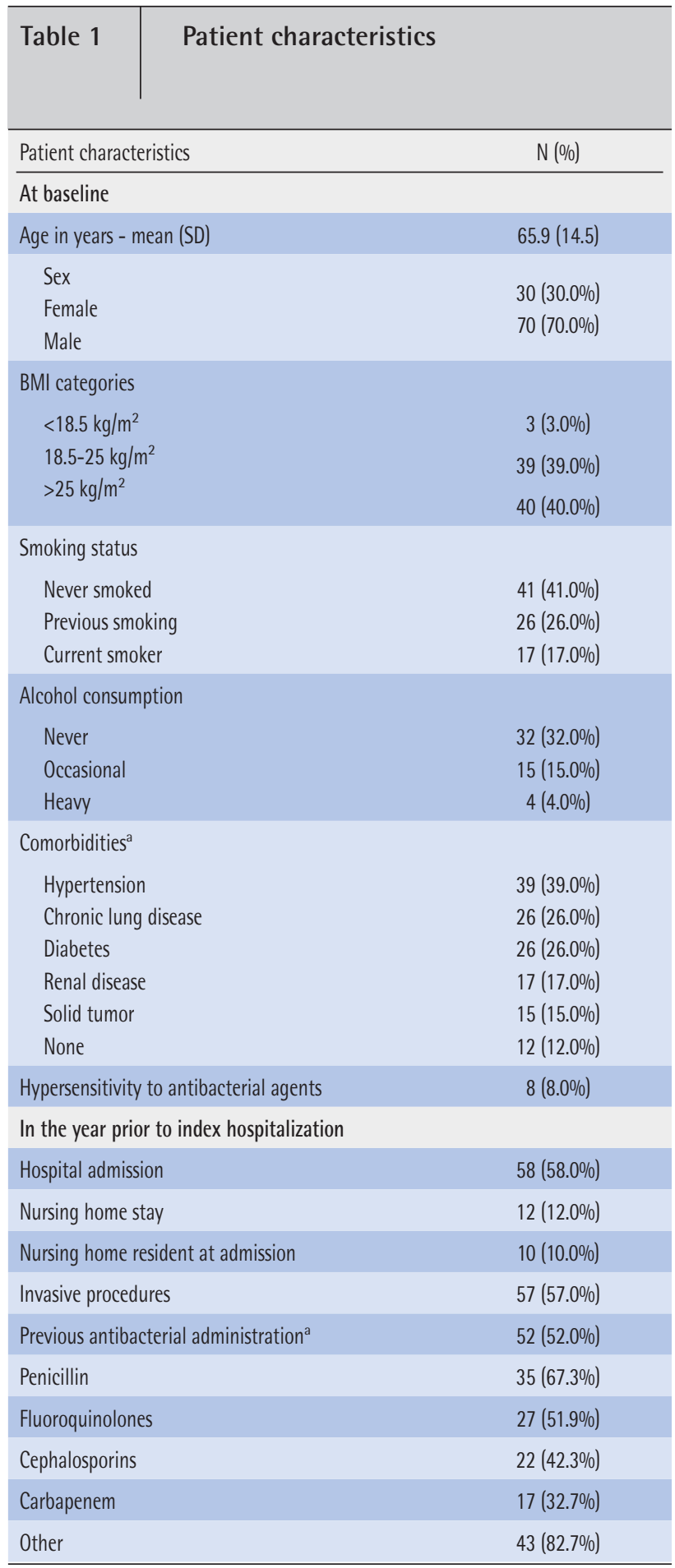

Multiresponse variable.

quired an ICU stay (Figure 3B) with a mean ICU LOS was 59.3 $(\mathrm{SD}=53.4)$ days (Figure 3C). Mean APACHE II score was 15.7 $(\mathrm{SD}=6.8$; Figure 3D).

Infection characteristics. The most common infection sites were HABP/VABP (47.0\%) and cUTI (42.0\%), while

\begin{tabular}{l|l}
\hline Table 1 & Patient characteristics (cont.) \\
& \\
\hline Patient characteristics & \\
\hline At admission & \\
Reason for admission & $36(36.0 \%)$ \\
Infection & $16(16.0 \%)$ \\
Respiratory & $15(15.0 \%)$ \\
Gastrointestinal & $7(7.0 \%)$ \\
Cardiovascular & $6(6.0 \%)$ \\
Trauma & $4(4.0 \%)$ \\
Neurological & $2(2.0 \%)$ \\
Metabolic/renal & $12(14.0 \%)$ \\
Other & \\
Ward of admission & $52(52.0 \%)$ \\
Medical & $29(29.0 \%)$ \\
ICU & $19(19.0 \%)$ \\
Surgical & \\
During ICU stay (n=50) & $15.7(6.8)$ \\
\hline APACHE II score (n=29) - mean (SD) & \\
\hline
\end{tabular}

${ }^{a}$ Multiresponse variable.

clAl was less common (6\%). 30\% of patients presented with secondary bacteremia (Table 2). Infections were mostly hospital-acquired $(71.0 \%)$ or healthcare-acquired $(21.0 \%)$, with K. pneumoniae (51.0\%) and P. aeruginosa (44.0\%) predominating. All pathogens were verified by culture, and E-test was the most commonly used (50.0\%) antibacterial susceptibility verification method. Of 106 isolates, 50 were analyzed using four types of carbapenemase tests: KPCNDM-OXA48 $(n=18)$, GeneExpert $(n=17)$, Hodge test $(n=15)$, and polymerase chain reaction (PCR) $(n=13)$ (Table 3 - note: since one isolate could be tested with more than one type of carbapenemase test, altogether 63 tests were performed on the 50 isolates). OXA was the most frequent confirmed carbapenemase type $(71.4 \%, n=42)$. As Figure 4 shows, between 50\%-96\% of tested K. pneumoniae isolates were non-susceptible to carbapenems, while all tested $P$. aeruginosa, $A$. baumannii, and $E$. coli isolates were non-susceptible to all carbapenems.

Clinical management characteristics. After culture collection, the most common antibacterial agents prescribed were carbapenems $(n=21,21.0 \%)$, penicillins $(n=15,15.0 \%)$, colistin $(n=6,6.0 \%)$, and sulfonamides $(n=6,6.0 \%)$. Immediately prior to the availability of susceptibility results, the most common antibacterial therapies prescribed were carbapenems $(n=41$, $41.0 \%)$, colistin $(n=20,20.0 \%)$, aminoglycosides $(n=19,19.0 \%)$, and penicillins $(n=19,19.0 \%)$. After test results were available, patients mostly received carbapenems $(n=41,41.0 \%)$, colistin $(n=28,28.0 \%)$, and aminoglycosides $(n=27,27.0 \%)$. Meropenem was the most frequently used carbapenem (95\% of all carbapenems prescribed). 
A

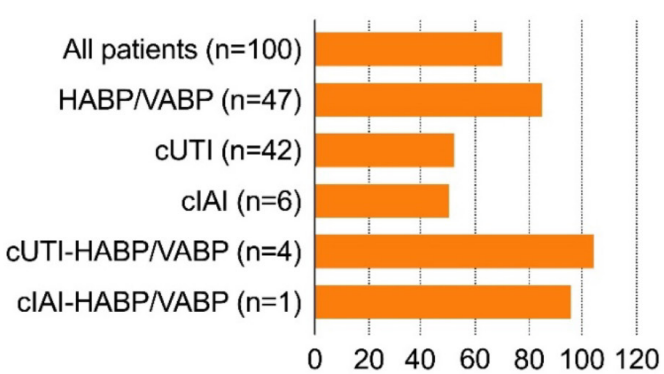

C

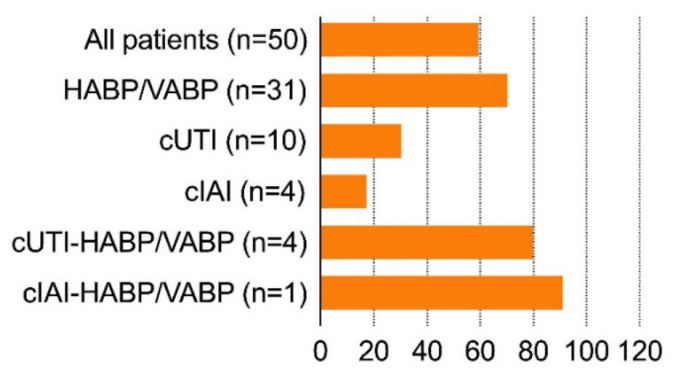

B

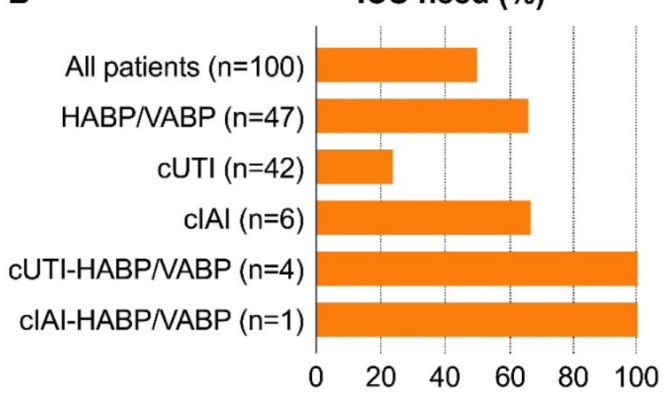

D

APACHE II score (points)

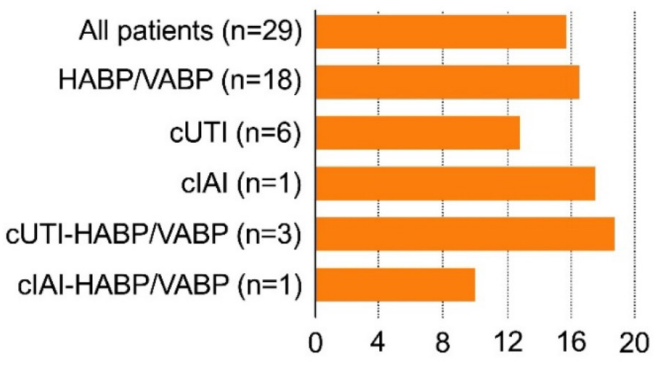

\begin{tabular}{l|l} 
Figure 3 & Index hospitalization characteristics. A: Length of stay for different infection
\end{tabular} sites (days). B: Intensive care unit need (percentage of all patients). C: Intensive care unit length of stay (days).

D: APACHE II score (points). Worst score if more than one admission. Based on limited data (44\% of patient data missing).

Data are presented as mean values.

APACHE II: Acute Physiology And Chronic Health Evaluation II; clAl: Complicated intra-abdominal infection; cUTI: Complicated urinary tract infection; HABP/VABP: Hospital-acquired bacterial pneumonia/ventilator-associated bacterial pneumonia; ICU: Intensive care unit; LOS: Length of stay; SD: Standard Deviation.

Figure 5 shows a Sankey diagram depicting the treatment pathways followed for patients who received a carbapenem $(n=57)$ during the follow-up period. For these patients, the most common practice immediately after culture collection was no treatment $(n=30)$, carbapenem monotherapy $(n=9)$, or carbapenem with one other antibacterial agent $(n=9)$. Receiving a carbapenem in combination with one other drug was the most common treatment path for patients both immediately before $(n=19)$ and after $(n=21)$ receiving the susceptibility results. The most frequent agents used in such combination with carbapenems were aminoglycosides $(n=9)$, colistin $(n=2)$ and linezolid $(n=2)$ immediately before and aminoglycosides $(n=11)$, colistin $(n=5)$, and glycopeptides $(n=2)$ after receiving the susceptibility results (data not shown). Additionally, colistin was frequently used in multiple-drug combinations. Overall, colistin was given to 6 patients immediately after culture collection, 20 patients before receipt and 28 patients after receipt of susceptibility results.

During hospitalization, the most common tests performed were biochemistry tests (92.0\% of patients), blood cell counts (84.0\%), urine cultures (68.0\%), blood cultures (67.0\%), and radiological investigations (57.0\%) (Table 4). Altogether 65.0\% of patients required invasive procedures, most commonly vascular catheterization (46.0\%), urinary catheterization (32.0\%), and mechanical ventilation (15.0\%).

Altogether $66.0 \%$ of patients achieved clinical cure within a mean of $19.9(S D=16.5)$ days after culture collection (Table 5). Severe sepsis/septic shock occurred in $35.0 \%$, with a mean duration of 20.3 ( $S D=18.2$ ) days. Mortality occurred in $35.0 \%$ of patients during hospitalization, and in $62.0 \%$ of patients by the time the chart review was performed. Within-hospitalization mortality happened at a mean of $147(S D=205)$ days from culture collection. The readmission rate at 6 -months post discharge was $47.7 \%(n=31)$, with the denominator being those patients who were alive at discharge.

\section{DISCUSSION}

The aim of our study was to characterize the realworld clinical management and outcomes of patients with complicated infections caused by CRGN pathogens in Spanish hospitals. Our main findings were that in the participating 


\begin{tabular}{|c|c|c|}
\hline Table 2 & \multicolumn{2}{|c|}{ Infection characteristics } \\
\hline Infection char & & $\mathrm{N}(\%)$ \\
\hline \multicolumn{3}{|l|}{ Infection site } \\
\hline HABP/NABF & & $47(47.0 \%)$ \\
\hline cUTI & & $42(42.0 \%)$ \\
\hline clAl & & $6(6.0 \%)$ \\
\hline cUTI and $\mathrm{H}$ & & $4(4.0 \%)$ \\
\hline $\mathrm{ClAl}$ and $\mathrm{HA}$ & & $1(1.0 \%)$ \\
\hline Secondary bac & & $30(30.0 \%)$ \\
\hline \multicolumn{3}{|c|}{ Place of acquiring infection ${ }^{a}$} \\
\hline Hospital-ac & & $71(71.0 \%)$ \\
\hline Healthcare- & & $21(21.0 \%)$ \\
\hline Community & & $8(8.0 \%)$ \\
\hline \multicolumn{3}{|c|}{ Pathogen isolated, per patient ${ }^{b}$} \\
\hline K. pneumol & & $51(51.0 \%)$ \\
\hline P. aerugino & & $44(44.0 \%)$ \\
\hline Other Enter & erales & $7(7.0 \%)$ \\
\hline A. bauman & & $3(3.0 \%)$ \\
\hline E. coli & & $1(1.0 \%)$ \\
\hline \multicolumn{3}{|c|}{ Pathogen verification ${ }^{b}$} \\
\hline Culture & & $100(100.0 \%)$ \\
\hline MALDI-TOF & & $19(19.0 \%)$ \\
\hline PCR & & $9(9.0 \%)$ \\
\hline Other & & $21(21.0 \%)$ \\
\hline \multicolumn{3}{|c|}{ Antibacterial susceptibility verification ${ }^{b}$} \\
\hline E-test & & $50(50.0 \%)$ \\
\hline Dilution & & $36(36.0 \%)$ \\
\hline Automated & icrobial susceptibility test & $32(32.0 \%)$ \\
\hline Disk diffusi & & $21(21.0 \%)$ \\
\hline \multicolumn{3}{|c|}{ Sample type $e^{b}$} \\
\hline Urine & & $58(58.0 \%)$ \\
\hline Sputum & & $37(37.0 \%)$ \\
\hline Blood & & $30(30.0 \%)$ \\
\hline BAL & & $17(17.0 \%)$ \\
\hline Peritoneal & & $6(6.0 \%)$ \\
\hline Pleural flui & & $2(2.0 \%)$ \\
\hline Other & & $2(2.0 \%)$ \\
\hline
\end{tabular}

aHospital-acquired: culture collected $=3$ days after admission; Healthcare-acquired: culture collected $<3$ days after admission with previous healthcare exposure; Community-acquired: culture collected $<3$ days after admission with no previous healthcare exposure.

${ }^{\mathrm{b}}$ Multiresponse variable.

BAL: Bronchoalveolar lavage; cIAl: Complicated intra-abdominal infection; cUTI: Complicated urinary tract infection; HABP/VABP: Hospital-acquired bacterial pneumonia/ventilator-associated bacterial pneumonia; MALDI-TOF: Matrix-assisted laser desorption ionization-time of flight mass spectrometry; PCR: Polymerase chain reaction.

\begin{tabular}{l|c}
\hline Table 3 & Carbapenemase testing procedures \\
& \\
\hline Characteristic & $\mathrm{N}(\%)$ \\
\hline \multicolumn{2}{|l|}{ Procedure(s) performed to determine carbapenemase type } \\
Not tested & $56(52.8 \%)$ \\
Tested & $50(47.2 \%)$ \\
KPC-NDM-OXA48 & $18(17.0 \%)$ \\
GeneExpert & $17(16.0 \%)$ \\
Hodge test & $15(14.1 \%)$ \\
PCR & $13(12.3 \%)$ \\
Carbapenemase type in confirmatory culture, positive tests & \\
OXA & $45(71.4 \%)$ \\
VIM & $3(4.8 \%)$ \\
Other & $15(23.8 \%)$ \\
\hline
\end{tabular}

asolates could be tested several times.

KPC: Klebsiella pneumoniae carbapenemase; NDM: New Delhi metallo-betalactamase; OXA: Oxacillinase type; PCR: Polymerase chain reaction; VIM: Verona Integron-Mediated metallo-beta-lactamase.

hospitals, about one in ten complicated infections involved CRGN pathogens. cUTI was found to have the most diverse carbapenem resistance profile in terms of carbapenemase production and $P$. aeruginosa and $A$. baumannii isolates showed the highest level of multi-carbapenem resistance. In addition, patients with CRGN infections in the retrospective cohort were most likely to acquire infections in hospitals or other healthcare settings, were most often suffering from $K$. pneumoniae or $P$. aeruginosa infections, and OXA was the most frequently detected carbapenemase type. Treatment escalation was common after susceptibility results became available, and the most common treatment was a carbapenem combined with another antibacterial agent. Clinical cure was achieved in two thirds of patients, but overall mortality was $62.0 \%$ by the end of the follow-up period.

The study sites reported a 9.3\% prior prevalence of CRGN pathogens for the assessed infection sites. While CRGN pathogens account for $3.2 \%$ of all healthcare-associated infections in Spain [6] complicated infections are more likely to be caused by multi-resistant pathogens as a result of the predisposing factors of the affected patient population and due to increased exposure of those patients to healthcare settings and invasive procedures [10]. This was evident in our study population, in which most patients were elderly and overweight, with frequent comorbidities. Previous episodes of hospitalization or nursing home stay, prior antibacterial treatment, and ICU admittance were all common, possibly playing a role in the emergence of antibacterial resistance, as these factors have been associated with resistant infections [11]. In particular, previous carbapenem therapy was observed in almost a third of patients, which is a commonly reported risk factor for the future acquisition of carbapenem-resistant infections, as previously described [12]. 


\section{K. pneumoniae}

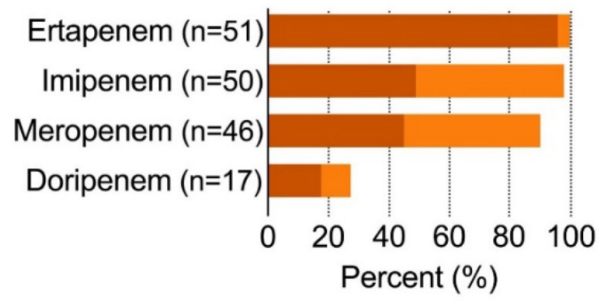

A. baumannii

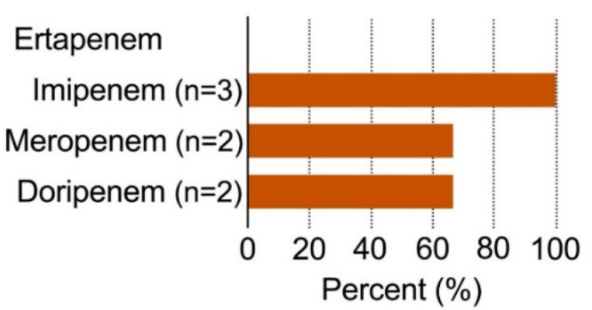

Enterobacterales

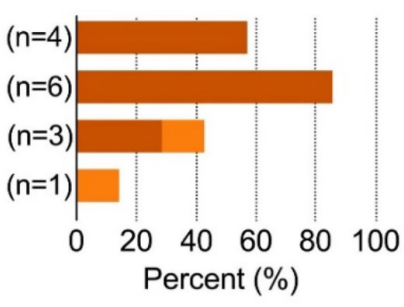

E. coli

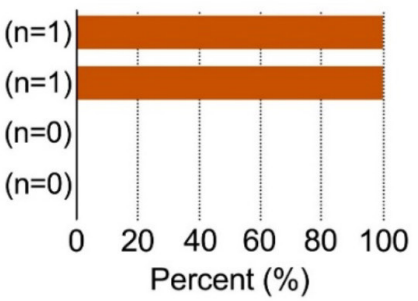

\section{P. aeruginosa}

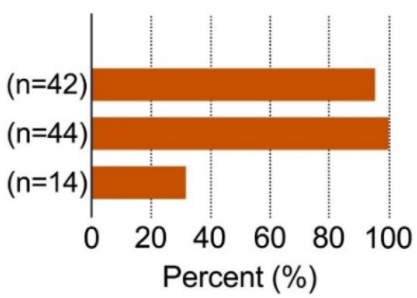

\section{Figure $4 \quad$ Antibiogram results}

Antibiogram information for confirmatory cultures. Colors represent the proportion of isolates which are susceptible/non-susceptible; the height of columns indicates the percentage of isolates tested. Ertapenem resistance is not shown for Pseudomonas and Acinetobacter isolates, since these pathogens are intrinsically resistant to this antibacterial agent.

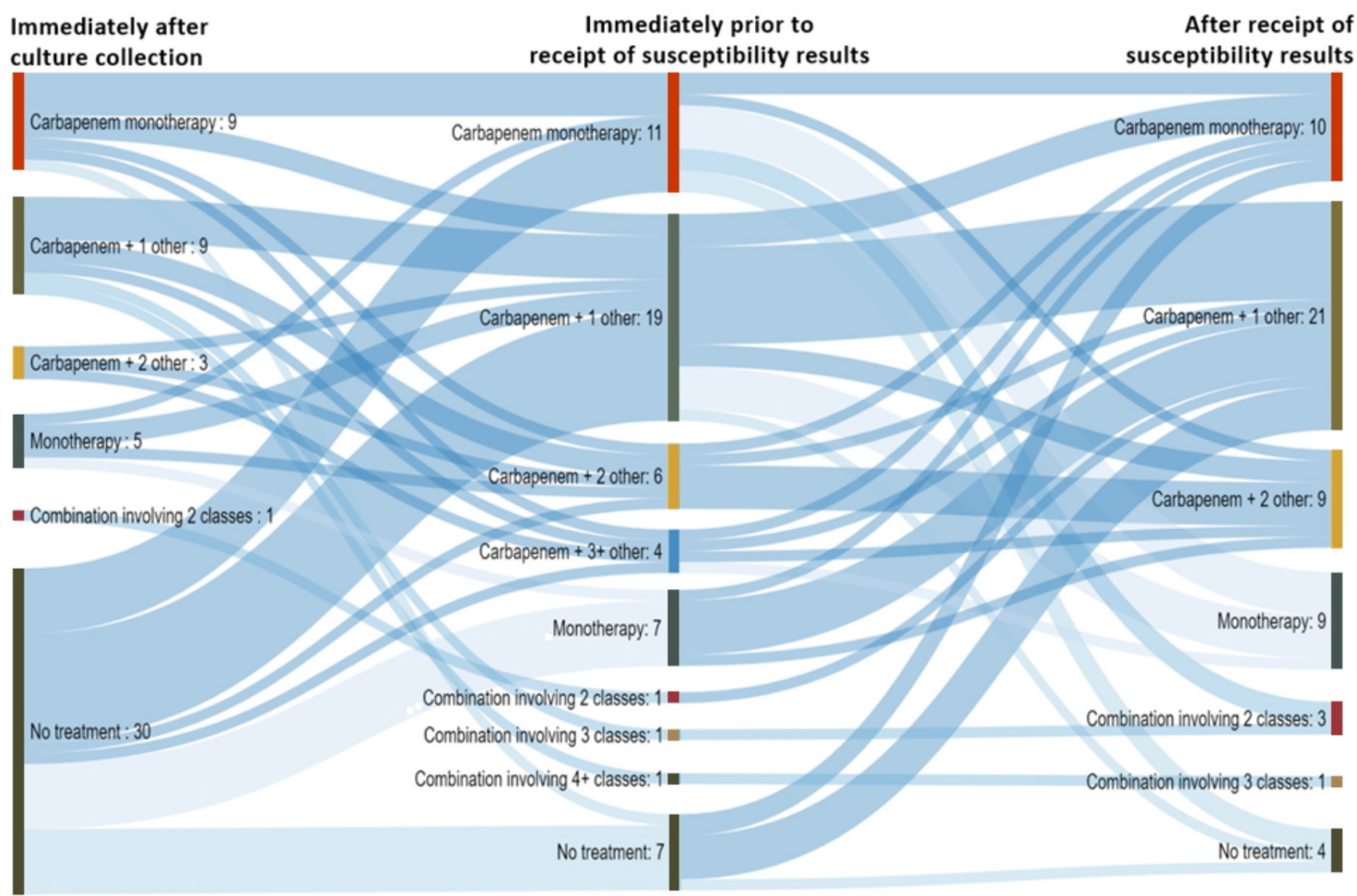

Figure 5 Sankey figure of treatment pathways for patients who received a carbapenem during observation period 


\begin{tabular}{|c|c|c|}
\hline \multirow{2}{*}{$\begin{array}{l}\text { Table } 4 \\
\text { Test or proced }\end{array}$} & \multicolumn{2}{|c|}{$\begin{array}{l}\text { Healthcare resource utilization: } \\
\text { Percentage of patients who underwent } \\
\text { diagnostic tests and/or invasive } \\
\text { procedures during index hospitalization }\end{array}$} \\
\hline & & $N(\%)$ \\
\hline \multicolumn{3}{|c|}{ Diagnostic tests during index hospitalization } \\
\hline Biochemi & & $92(92.0 \%)$ \\
\hline Blood cel & & $84(84.0 \%)$ \\
\hline Urine cul & & $68(68.0 \%)$ \\
\hline Blood cul & & $67(67.0 \%)$ \\
\hline Radiogra & & $57(57.0 \%)$ \\
\hline Sputum a & & $44(44.0 \%)$ \\
\hline CT & & $28(28.0 \%)$ \\
\hline Other dia & & $19(19.0 \%)$ \\
\hline ECG & & $18(18.0 \%)$ \\
\hline Bronchos & & $15(15.0 \%)$ \\
\hline MRI & & $5(5.0 \%)$ \\
\hline \multicolumn{3}{|c|}{ Invasive procedures during index hospitalization } \\
\hline Vascular & & $46(46.0 \%)$ \\
\hline Urinary c & & $32(32.0 \%)$ \\
\hline Mechanic & ation & $15(15.0 \%)$ \\
\hline Surgery $(i$ & ical drainage) & $7(7.0 \%)$ \\
\hline Endoscop & & $6(6.0 \%)$ \\
\hline Percutane & nage & $5(5.0 \%)$ \\
\hline Cardiac c & ation & $1(1.0 \%)$ \\
\hline \multicolumn{3}{|c|}{ Diagnostic tests following index hospitalization } \\
\hline Biochemi & & $5(5.0 \%)$ \\
\hline Blood cel & & $5(5.0 \%)$ \\
\hline Urine cul & & $5(5.0 \%)$ \\
\hline Blood cul & & $3(3.0 \%)$ \\
\hline Radiogra & & $5(5.0 \%)$ \\
\hline CT & & $2(2.0 \%)$ \\
\hline Other dia & & $2(2.0 \%)$ \\
\hline Sputum a & & $1(1.0 \%)$ \\
\hline
\end{tabular}

Table shows diagnostic tests and invasive procedures performed during index hospitalization and diagnostic tests in the follow up period for patients who have reported at minimum one diagnostic test related to infection treatment from 6 months after discharge (data regarding invasive procedures in this period was not available). CT: computed tomography, ECG electrocardiogram, MRI: magnetic resonance imaging.

Most CRGN infections were acquired in a healthcare-related setting and were most often caused by or. The high level of CR K. pneumoniae is especially noteworthy, since this pathogen is frequently associated with healthcare-associated outbreaks and has an almost exclusively healthcare-associated pattern of occurrence [2]. While the prevalence of this pathogen in Spain is lower than the European average, the ECDC and local sources have identified a recent alarming increase $[2$,

\begin{tabular}{|c|c|c|}
\hline Table 5 & Outcomes & \\
\hline \multicolumn{2}{|l|}{ Characteristic } & $\mathrm{N}(\%)$ \\
\hline \multicolumn{2}{|c|}{ Clinical cure achieved ${ }^{\mathrm{a}}$} & $66(66.0 \%)$ \\
\hline \multicolumn{2}{|c|}{ Time to clinical cure from culture collection - days - mean (SD) } & $19.9(16.5)$ \\
\hline \multicolumn{2}{|c|}{ Severe sepsis/septic shock } & $35(35.0 \%)$ \\
\hline \multicolumn{2}{|c|}{ Duration of severe sepsis/septic shock - days - mean (SD) } & $20.3(18.2)$ \\
\hline \multicolumn{2}{|c|}{ Mortality during index hospitalization } & $35(35.0 \%)$ \\
\hline \multicolumn{2}{|c|}{ Time to discharge from culture collection - days - mean (SD) } & $44.6(53.8)$ \\
\hline \multicolumn{2}{|c|}{ Time to ICU discharge since culture collection - days - mean (SD) } & $17.2(33.5)$ \\
\hline \multicolumn{2}{|c|}{ Time to death since culture collection - days - mean (SD) } & $147.0(205.0)$ \\
\hline \multicolumn{2}{|c|}{$\begin{array}{l}\text { Time to readmission from discharge following index } \\
\text { hospitalization - days - mean (SD) }\end{array}$} & $54(44.0)$ \\
\hline \multicolumn{2}{|c|}{ Readmission rate at 30 days $^{c}$} & $8(12.3 \%)$ \\
\hline \multicolumn{2}{|c|}{ Readmission date at 60 days $^{c}$} & $31(47.7 \%)$ \\
\hline \multicolumn{2}{|c|}{ Overall mortality (at chart review) } & $62(62 \%)$ \\
\hline \multicolumn{3}{|c|}{ Cause of death } \\
\hline \multicolumn{2}{|l|}{ Unknown } & $10(16.1 \%)$ \\
\hline \multicolumn{2}{|c|}{ Infection-related } & $18(29.0 \%)$ \\
\hline \multicolumn{2}{|l|}{ Other } & $34(54.8 \%)$ \\
\hline
\end{tabular}

${ }^{\mathrm{a}}$ As defined by complete resolution of all signs and symptoms of infection.

${ }^{\text {b}}$ Time to readmission since discharge was calculated for all patients who reported a hospitalization up to 6 months after hospital discharge. Those patients with missing information about status (alive or deceased) at 6 months following hospital discharge were removed from the population used to assess readmission, in order to avoid a potential bias to higher hospitalization rates.

'Readmission rate was calculated using the number of alive patients at discharge as denominator.

13]. A similar upward trend has not been noted for $P$. aeruginosa, a ubiquitous and opportunistic microbe, which still remains one of the most common CRGN pathogens both across the EU and within Spain [2]. The adaptive antibiotic resistance of $P$. aeruginosa mainly includes biofilm mediated resistance and formation of multidrug-tolerant persister cells. Nonetheless, in our study we focused the analysis on carbapenemase. Other Enterobacterales spp. contributed to infection in only $10 \%$ of the patients in our study population, a lower rate than that observed in other Southern and Eastern European countries [2].

All isolates were identified via culture, potentially signifying that rapid diagnostic procedures may decrease diagnostic time and improve clinical decision making. Antibacterial susceptibility was most often verified by E-test, dilution, and automated antimicrobial susceptibility testing methods. Carbapenemase tests, which are recommended by CLSI guidelines [14] whenever the minimum inhibitory concentrations of carbapenems are 2-4 mg/L, were used quite frequently in our study, especially for $P$. aeruginosa and $K$. pneumoniae isolates. The 
most frequent carbapenemase types obtained in the confirmatory culture, following carbapenemase testing, were OXA-type, which is a resistance mechanism recognized as a public health threat worldwide [15] and in Spain in particular [13]. Based on the results of our study, an improvement in the frequency and quality of testing, e.g. more frequent use of PCR and MALDI-TOF based methods for isolate identification $[16,17]$ and extensive carbapenemase identification could be an important tool to improve optimal targeted therapy selection in Spanish hospitals.

Treatment pattern analysis in our study with 3 different cross-sectional timepoints (at confirmatory culture i.e. empiric, prior to susceptibility results, and after susceptibility results) gives a unique real-world insight into the current management of CRGN infections in Spain. We found that carbapenems were frequently used to treat cUTI, CIAI, HABP/VABP caused by CRGN pathogens. Carbapenem use, particularly in combination with other agents, persisted after confirmation of carbapenem resistance. The high prevalence of carbapenem use throughout the three timepoints of the study supports their important role as frequently used antibacterial agents for GN infections, despite their inappropriateness for this patient population. Our results indicated treatment escalation, with combination therapies occurring more frequently as patients progressed throughout the three timepoints, perhaps due to clinical deterioration occurring while the patient received an inappropriate empiric therapy or reflecting the receipt of susceptibility test data confirming its inappropriateness. Colistin was the second most frequently received antibacterial agent after meropenem, despite several known disadvantages - including difficulty in obtaining optimal dosing, high renal toxicity, and unclear susceptibility testing due to the absence of agreed breakpoints [18].

Tigecycline, fosfomycin, and aztreonam are widely used in Spain. However, a large proportion of critically ill patients with multidrug-resistant $\mathrm{GN}$ infection are treated with tigecycline and do not achieve an appropriate pharmacokinetic and pharmacodynamic value. Even, tigecycline seems to be associated with hepatobiliary disorders in the critically ill population [19]. Fosfomycin has a very unique mechanism of action against Gram-positive and GN bacteria. Cross-resistance seems uncommon. However, fosfomycin should be used in combination in patients with severe infections to avoid selecting resistant mutations [20].

The considerable proportion of patients still not receiving antibacterial agents or only receiving carbapenem monotherapy both before and after susceptibility results (despite this being a patient population with carbapenem-resistant infections), could indicate the difficulty of selecting adequate empirical or effective targeted antibacterial therapies. These prescribing behaviours might contribute to some of the unfavourable outcomes seen in our study and highlight the need for new treatment options in this clinical population. Novel $\beta$-lactam $/ \beta$-lactamase inhibitor combinations, namely meropenem/vaborbactam, ceftazidime/avibactam, ceftolozane/tazobactam, and imipenem/cilastatin/relebactam are promising options in certain CRGN infections due to their ability to overcome carbapenem resistance, although resistance has already been reported to some of these novel agents [21]. The use of these novel drugs in Spain was still limited at the time of our study.

The EU-CARE study design permits a robust evaluation of clinical outcomes of the index hospitalization associated with complicated infections caused by CRGN pathogens. We found that only $66.0 \%$ of patients experienced clinical cure, which underlines the difficult clinical challenge of treating CRGN infections. Furthermore, outcomes for study subjects were generally poor, with $35.0 \%$ mortality during index hospitalization and more than $60.0 \%$ overall mortality by the end of the follow-up period. This is higher than the expected 24.0\% mortality rate estimated for patients with a mean APACHE II score of 15.7 points [22] but it is in line with the results of a recent meta-analysis reporting $32.0 \%$ to $88.4 \%$ mortality for different CRGN infections at varying time points [23].

The financial burden of CRGN infections has been well described and is associated with costs for drugs, hospital stay, and diagnostic procedures [24]. The direct and indirect economic burden is one of the main criteria that lead to CRGN infections being considered a major healthcare issue for Spain [25]. The EU-CARE study corroborates these data, as length of stay, ICU need, and readmission rates were all considerable, pointing to substantial healthcare burden when treating these types of infections.

The EU-CARE study had several limitations. First, our sampling frame of the participating hospitals may not be representative of the country, given the small number of participating sites and that they were enrolled by convenience sampling as opposed to random selection. Furthermore, the retrospective chart review design might have contributed to the under-recording of information and including patients with the same pathogen profile due to the possible clustering of hospital-acquired pathogens in a particular hospital site. This limitation might provide a potential explanation for the high prevalence of $K$. pneumoniae in our cohort. The study eligibility criteria allowed for any CRGN pathogen-associated complicated infection to be included, and although this reflects real life practice (i.e. hospitals must treat varying causative pathogen and infection sites), outcome parameters may not be comparable between different pathogens or infections sites within our cohort and may prevent comparison of our data with data from prior studies focusing on a specific infection site and/or specific bacterial species. It is also important to note that since the completion of the study, EUCAST has implemented changes in their definitions of susceptibility testing categories $S, I$, and R [26]. Since our study included both resistant and intermediate agents as "non-susceptible", some included cases may now not qualify as CR given the new definitions.

In conclusion, the results of the EU-CARE study reflect the high burden and unmet need associated with the management of complicated infections attributable to CRGN pathogens in Spain. Our results also indicate that carbapenems are com- 
monly prescribed empirically and remain in use even after the verification of carbapenem resistance, though often in combination with potentially synergistic, but sometimes toxic agents such as colistin. The observed treatments given to this study cohort may indicate a lack of safe and effective treatment options for this high need patient population. Poor outcomes and increased healthcare resource use lead to a considerable human and financial burden to healthcare settings and further reinforces the urgent need for enhanced clinical management of difficult-to-treat CRGN infections.

\section{ACKNOWLEDGEMENTS}

The authors would like to thank the medical communications agency EpiConsult for their assistance in the preparation of the manuscript, tables, and figures.

\section{FUNDING}

Funding for this research was provided by Merck Sharp \& Dohme Corp., a subsidiary of Merck \& Co., Inc., Kenilworth, NJ, USA.

\section{CONFLICTS OF INTEREST}

Dr Ferrer reports personal fees from Bazter, Estor, Grifols, MSD, Pfizer, and Shionogi; and grants and personal fees from Beckton-Dickinson and Thermofisher, outside the submitted work. Dr Salavert reports has previously participated as advisory board member for MSD (receiving non-financial support) and Pfizer. Also reports he has participated as speaker for Angelini, Gilead (receiving non-financial support), Janssen, MSD and Pfizer outside the submitted work.

Drs Diaz-Regañón, López and Lozano are employees in MSD, Spain. Dr Gómez-Ulloa reports other from Merck \& Co. Inc, during the conduct of the study. Dr McCann reports personal fees from Merck \& Co., Inc., outside the submitted work. No other disclosures were reported.

\section{REFERENCES}

1. Marston HD, Dixon DM, Knisely JM, Palmore TN, Fauci AS. Antimicrobial Resistance. JAMA 2016; 316(11): 1193-204. DOI: 10.1001/ jama.2016.11764

2. European Centre for Disease Prevention and Control. Surveillance of antimicrobial resistance in Europe. 2017. Available from: https:// www.ecdc.europa.eu/en/publications-data/surveillance-antimicrobial-resistance-europe-2017 (accessed June 1st 2021).

3. WHO. World Health Organization. Antimicrobial resistance: Global Report on Surveillance. 2014. Available from: https://www.who.int/ antimicrobial-resistance/publications/surveillancereport/en/ (accessed June 1st 2021).

4. Nordmann P, Poirel L. Epidemiology and Diagnostics of Carbapenem Resistance in Gram-negative Bacteria. Clin Infect Dis: an offi- cial publication of the Infectious Diseases Society of America 2019; 69(Suppl 7): S521-s8. D0I: 10.1093/cid/ciz824

5. Paul M, Daikos GL, Durante-Mangoni E, Yahav D, Carmeli Y, Benattar YD, et al. Colistin alone versus colistin plus meropenem for treatment of severe infections caused by carbapenem-resistant Gram-negative bacteria: an open-label, randomised controlled trial. Lancet Infect Dis 2018; 18(4): 391-400. DOI: 10.1016/S14733099(18)30099-9.

6. Cantón R, Huarte R, Morata L, Trillo-Mata JL, Muñoz R, González J, et al. Determining the burden of infectious diseases caused by carbapenem-resistant gram-negative bacteria in Spain. Enferm Infecc Microbiol Clin 2020. DOI: 10.1016/j.eimc.2020.04.009.

7. Grau S, Fondevilla E, Echeverría-Esnal D, Alcorta A, Limon E, Gudiol $F$, et al. Widespread increase of empirical carbapenem use in acute care hospitals in Catalonia, Spain. Enferm Infecc Microbiol Clin 2019; 37(1): 36-40. DOI: 10.1016/j.eimc.2018.03.003.

8. Peleg AY, Hooper DC. Hospital-acquired infections due to gram-negative bacteria. N Engl J Med 2010; 362(19): 1804-13. DOI: 10.1056/NEJMra0904124.

9. Testing. TECoAS. Breakpoint tables for interpretation of MICs and zone diameters. 2017. Available from:https://www.eucast.org/ fileadmin/src/media/PDFs/EUCAST_files/Breakpoint_tables/v_7.1_ Breakpoint_Tables.pdf (accessed June 1st 2021).

10. Martín-Loeches I, Diaz E, Vallés J. Risks for multidrug-resistant pathogens in the ICU. Curr Opin Crit Care 2014; 20(5): 516-24. DOI: 10.1097/MCC.0000000000000124

11. Segagni Lusignani L, Presterl E, Zatorska B, Van den Nest M, Diab-Elschahawi M. Infection control and risk factors for acquisition of carbapenemase-producing enterobacteriaceae. A 5 year (20112016) case-control study. Antimicrob Resist Infect Control 2020; 9(1): 18. DOI: 10.1186/s13756-019-0668-2

12. Katchanov J, Asar L, Klupp EM, Both A, Rothe C, König C, et al. Carbapenem-resistant Gram-negative pathogens in a German university medical center: Prevalence, clinical implications and the role of novel $\beta$-lactam/ $\beta$-lactamase inhibitor combinations. PloS One 2018; 13(4): e0195757. DOI: 10.1371/journal.pone.0195757.

13. Hernández-García M, Pérez-Viso B, Carmen Turrientes M, Díaz-Agero C, López-Fresneña N, Bonten M, et al. Characterization of carbapenemase-producing Enterobacteriaceae from colonized patients in a university hospital in Madrid, Spain, during the R-GNOSIS project depicts increased clonal diversity over time with maintenance of high-risk clones. J Antimicrob Chemother 2018; 73(11): 3039-43. DOI: $10.1093 / j a c / d k y 284$.

14. Weinstein MP, Patel JB, Campeau S, Eliopoulus GM, Galas MF, Humphries RM, et al. CLSI performance standards for antimicrobial susceptibility testing. CLSI supplement M100. 28th ed.: Clinical and Laboratory Standards Institute, PA, USA, 2018.

15. Carrër A, Poirel L, Yilmaz M, Akan OA, Feriha C, Cuzon G, et al. Spread of OXA-48-encoding plasmid in Turkey and beyond. Antimicrob Agents Chemother 2010; 54(3): 1369-73. DOI: 10.1128/ AAC.01312-09.

16. Oviaño M, Sparbier K, Barba MJ, Kostrzewa M, Bou G. Universal protocol for the rapid automated detection of carbapenem-resistant 
Gram-negative bacilli directly from blood cultures by matrix-assisted laser desorption/ionisation time-of-flight mass spectrometry (MALDI-TOF/MS). Int J Antimicrob Agents 2016; 48(6): 655-60. DOI: 10.1016/j.ijantimicag.2016.08.024.

17. Shields RK, Clancy CJ, Hao B, Chen L, Press EG, Lovine NM, et al. Effects of Klebsiella pneumoniae carbapenemase subtypes, extended-spectrum $\beta$-lactamases, and porin mutations on the in vitro activity of ceftazidime-avibactam against carbapenem-resistant $\mathrm{K}$. pneumoniae. Antimicrob Agents Chemother 2015; 59(9): 5793-7. DOI: 10.1128/AAC.00548-15.

18. EUCAST. Problems with colistin susceptibility testing and several commercially available products. 2016. Available from: https://eucast.org/ast_of_bacteria/warnings/ (accessed June 1st 2021).

19. Ruiz J, Ramirez $P$, Villarreal $E$, Gordon $M$, Sánchez MA, Martín $M$, et al. Effect of pharmacokinetic/pharmacodynamic ratio on tigecycline clinical response and toxicity in critically ill patients with multidrug-resistant Gram-negative infections. SAGE Open Med. 2020 Sep 18;8:2050312120958897. doi: 10.1177/2050312120958897.

20. Múñez Rubio E, Ramos Martínez A, Fernández Cruz A. Fosfomycin in antimicrobial stewardship programs. Rev Esp Quimioter. 2019 May;32 Suppl 1(Suppl 1):62-66. PMID: 31131594; PMCID: PMC6555160.

21. Castanheira M, Mills JC, Farrell DJ, Jones RN. Mutation-driven $\beta$-lactam resistance mechanisms among contemporary ceftazidime-nonsusceptible Pseudomonas aeruginosa isolates from U.S. hospitals. Antimicrob Agents Chemother 2014; 58(11): 6844-50. DOI: 10.1128/AAC.03681-14.

22. Knaus WA, Draper EA, Wagner DP, Zimmerman JE. APACHE II: a severity of disease classification system. Crit Care Med 1985; 13(10): 818-29. PMID: 3928249

23. Martin A, Fahrbach K, Zhao Q, Lodise T. Association Between Carbapenem Resistance and Mortality Among Adult, Hospitalized Patients With Serious Infections Due to Enterobacteriaceae: Results of a Systematic Literature Review and Meta-analysis. Open Forum Infect Dis 2018; 5(7): ofy150. D0I: 10.1093/ofid/ofy150.

24. Vargas-Alzate CA, Higuita-Gutiérrez LF, López-López L, Cienfuegos-Gallet AV, Jiménez Quiceno JN. High excess costs of infections caused by carbapenem-resistant Gram-negative bacilli in an endemic region. Int J Antimicrob Agents 2018; 51(4): 601-7. PMID: 29277527

25. Alfonso JL, Cantón R, Ferrer R. Determining the burden of carbapenem-resistant Gram-negative infections in Spain by multi-criteria decision analysis (MCDA). Value Health 2019; 22: S656-S7.

26. EUCAST. The European Committee on Antimicrobial Susceptibility Testing. S, I and R definitions. 2019. Available from: https://www. eucast.org/newsiandr/ (accessed June 1st 2021). 